\title{
Phase-shifting algorithms for electronic speckle pattern interferometry
}

\author{
Chih-Cheng Kao, Gym-Bin Yeh, Shu-Sheng Lee, Chih-Kung Lee, Ching-Sang Yang, \\ and Kuang-Chong Wu
}

\begin{abstract}
A set of innovative phase-shifting algorithms developed to facilitate metrology based on electronic speckle pattern interferometry (ESPI) are presented. The theory of a phase-shifting algorithm, called a $(5,1)$ algorithm, that takes five phase-shifted intensity maps before a specimen is deformed and one intensity map after a specimen is deformed is presented first. Because a high-speed camera can be used to record the dynamic image of the specimen, this newly developed algorithm has the potential to retain the phase-shifting capability for ESPI in dynamic measurements. Also shown is an algorithm called a $(1,5)$ algorithm that takes five phase-shifted intensity maps after the specimen is deformed. In addition, a direct-correlation algorithm was integrated with these newly developed $(5,1)$ or $(1,5)$ algorithms to form DC- $(5,1)$ and DC- $(1,5)$ algorithms, which are shown to improve significantly the quality of the phase maps. The theoretical and experimental aspects of these two newly developed techniques, which can extend ESPI to areas such as high-speed dynamic measurements, are examined in detail. (c) 2002 Optical Society of America
\end{abstract}

OCIS codes: $110.6150,120.5050,120.6160,120.6650$.

\section{Introduction}

Since electronic speckle pattern interferometry (ESPI) was invented in the 1970s, algorithms and configurations to simplify measurement procedures have been continually proposed. ${ }^{1-3}$ A phase-shifting technique, ${ }^{4,5}$ which has been one of the most important achievements among these approaches, provides a simple and easy approach to facilitating use of ESPI. While implementing phase-shifting techniques for ESPI, we introduce the phase modulation of a known magnitude to arrive at a series of intensity maps so as to compute the wrapped phase maps. The displacement data of interest can then be obtained by phase-unwrap techniques to process the phase map. The most serious bottleneck that prevents easy implementation of the above-mentioned approach has been erroneous data recovered from a noisy phase map. To overcome this bottleneck, a set of newly developed metrology and signal-processing

The authors are with the Institute of Applied Mechanics, National Taiwan University, Taipei 106, Taiwan. C.-K. Lee's e-mail address is cklee@mems.iam.ntu.edu.tw.

Received 19 October 2000; revised manuscript received 18 July 2001.

0003-6935/02/010046-09\$15.00/0

(C) 2002 Optical Society of America algorithms is integrated within this paper. The first of the two sets of methods consists of first the $(5,1)$ algorithm and second the $(1,5)$ algorithm. The difference between these two algorithms is whether the five-step phase-shifting algorithm is adopted before or after a specimen is deformed. The second of the two newly developed signal-processing algorithms further integrates the direct-correlation algorithm ${ }^{6-8}$ with either the $(5,1)$ or the $(1,5)$ algorithms to improve the quality of the phase maps. These two sets of algorithms, called the DC- $(5,1)$ algorithm and the DC- $(1,5)$ algorithm, are presented in detail.

\section{Theory}

To implement ESPI, intensity maps must be recorded before and after a specimen is deformed. Before deformation the intensity $I_{B}$ formed by interference between the object and the reference beams is

$$
I_{B}=I_{1}+I_{2}+2\left(I_{1} I_{2}\right)^{1 / 2} \cos \left(\Phi_{R}-\Phi_{B}\right),
$$

where $I_{1}$ and $I_{2}$ are the intensities of the object and the reference beams, respectively, and $\Phi_{R}$ and $\Phi_{B}$ are the phases of the speckle pattern of the reference light beam and the undeformed sampling light beam. The reference light beam and the undeformed sampling light beam are coherent in the local region of each independent speckle and not in the whole area. More specifically, the interference speckle pattern 
shown in Eq. (1) varies significantly for each individual speckle region. The intensity of the interference $I_{A}$ generated after deformation becomes

$$
I_{A}=I_{1}+I_{2}+2\left(I_{1} I_{2}\right)^{1 / 2} \cos \left(\Phi_{R}-\Phi_{B}+\Delta \Phi\right),
$$

where $\Delta \Phi$ is the phase change due to the object deformation. Secondary fringes are calculated by $I_{F}$, obtained by subtracting the two intensities mentioned above as

$$
I_{F}=I_{B}-I_{A} .
$$

Since there are a total of four unknowns, $I_{1}, I_{2},\left(\Phi_{R}-\right.$ $\Phi_{B}$ ), and $\Delta \Phi$, in Eq. (3) the phase difference $\Delta \Phi$ of interest, which contains the information of the displacement, cannot be retrieved directly. To overcome this difficulty, a phase-shifting technique ${ }^{4,5}$ has been introduced into ESPI. With the recording of the intensity maps before and after deformation, the phase of the speckle pattern in either state can be computed with the procedures described below. In a traditional five-step algorithm, ${ }^{5}$ five predesignated phase changes, $-2 \beta,-\beta, 0,+\beta,+2 \beta$, were introduced into each intensity map and a total of five intensity maps were recorded, that is

$$
\begin{aligned}
& I_{a}=I_{1}+I_{2}+2\left(I_{1} I_{2}\right)^{1 / 2} \cos \left(\Phi_{R}-\Phi_{B}-2 \beta\right), \\
& I_{b}=I_{1}+I_{2}+2\left(I_{1} I_{2}\right)^{1 / 2} \cos \left(\Phi_{R}-\Phi_{B}-\beta\right), \\
& I_{c}=I_{1}+I_{2}+2\left(I_{1} I_{2}\right)^{1 / 2} \cos \left(\Phi_{R}-\Phi_{B}\right), \\
& I_{d}=I_{1}+I_{2}+2\left(I_{1} I_{2}\right)^{1 / 2} \cos \left(\Phi_{R}-\Phi_{B}+\beta\right), \\
& I_{e}=I_{1}+I_{2}+2\left(I_{1} I_{2}\right)^{1 / 2} \cos \left(\Phi_{R}-\Phi_{B}+2 \beta\right) .
\end{aligned}
$$

The phase of the speckle pattern in the undeformed state can be calculated as

$$
\tan \left(\Phi_{R}-\Phi_{B}\right)=\frac{[1-\cos (2 \beta)]}{\sin (\beta)} \frac{\left(I_{b}-I_{d}\right)}{\left(2 I_{c}-I_{a}-I_{e}\right)} .
$$

When the phase change $\beta$ is set as $\pi / 2$, Eq. (5) becomes

$$
\tan \left(\Phi_{R}-\Phi_{B}\right)=\frac{2\left(I_{b}-I_{d}\right)}{\left(2 I_{c}-I_{a}-I_{e}\right)} .
$$

The same five-step algorithm can calculate similarly the phase of the speckle pattern in the deformed state. Five intensity maps recorded at the deformed state can be written as

$$
\begin{aligned}
& I_{a}^{*}=I_{1}+I_{2}+2\left(I_{1} I_{2}\right)^{1 / 2} \cos \left(\Phi_{R}-\Phi_{B}+\Delta \Phi-2 \beta\right), \\
& I_{b}{ }^{*}=I_{1}+I_{2}+2\left(I_{1} I_{2}\right)^{1 / 2} \cos \left(\Phi_{R}-\Phi_{B}+\Delta \Phi-\beta\right), \\
& I_{c}{ }^{*}=I_{1}+I_{2}+2\left(I_{1} I_{2}\right)^{1 / 2} \cos \left(\Phi_{R}-\Phi_{B}+\Delta \Phi\right), \\
& I_{d}{ }^{*}=I_{1}+I_{2}+2\left(I_{1} I_{2}\right)^{1 / 2} \cos \left(\Phi_{R}-\Phi_{B}+\Delta \Phi+\beta\right), \\
& I_{e}^{*}=I_{1}+I_{2}+2\left(I_{1} I_{2}\right)^{1 / 2} \cos \left(\Phi_{R}-\Phi_{B}+\Delta \Phi+2 \beta\right),
\end{aligned}
$$

The phase of the speckle pattern measured from the deformed state is thus

$$
\tan \left(\Phi_{R}-\Phi_{B}+\Delta \Phi\right)=\frac{2\left(I_{b}{ }^{*}-I_{d}{ }^{*}\right)}{\left(2 I_{c}^{*}-I_{a}{ }^{*}-I_{e}{ }^{*}\right)} .
$$

Traditionally the phase difference $\Delta \Phi$ was obtained by subtracting Eq. (8) from Eq. (6), i.e.,

$$
\begin{aligned}
\Delta \Phi= & \tan ^{-1}\left[\frac{2\left(I_{b}^{*}-I_{d}^{*}\right)}{\left(2 I_{c}^{*}-I_{a}^{*}-I_{e}^{*}\right)}\right] \\
& -\tan ^{-1}\left[\frac{2\left(I_{b}-I_{d}\right)}{\left(2 I_{c}-I_{a}-I_{e}\right)}\right] .
\end{aligned}
$$

The phase difference $\Delta \Phi$, which may jump by $2 \pi$ from one point to the next, is thus called the wrapped phase map. To overcome this discontinuity so as to retrieve the true (unwrapped) phase map that relates to specimen deformation, a phase-unwrapping technique such as the discreet cosine transform (DCT) was introduced. ${ }^{9-13}$ Once the unwrapped phase map is retrieved, the specimen deformation can be easily calculated by multiplying a constant to the unwrapped phase map. The constant can be determined by the optical configuration and represents the relationship between the phase and the specimen deformation. ${ }^{1-3}$

\section{3. (1,5) and (5,1) Algorithms}

In the traditional phase-shifting techniques mentioned above, a large number of computations are required because two independent rounds of phaseshifting processes must be executed. Two innovative phase-shifting algorithms, the $(5,1)$ algorithm and the $(1,5)$ algorithm, are proposed to reduce computation time. The difference between these two algorithms is whether phase-shifting steps are performed in the undeformed or the deformed state. More specifically, the $(5,1)$ algorithm indicates that the five-step phase-shifting technique is performed in the undeformed state and a single intensity phase is measured in the deformed state. In contrast, the $(1,5)$ algorithm indicates that the five-step phaseshifting technique is performed in the deformed state. It is clear from the discussions above that the $(5,1)$ algorithm has the potential to be implemented for dynamic ESPI measurements because only a single intensity map in the deformed state is needed and a high-speed camera can be used to record the dynamic deformations of the specimen. That is, signal processing for the $(5,1)$ algorithm can be executed after all the dynamic deformation intensity maps of the specimen are recorded by the high-speed camera and can, as such, be executed at the same pace as the signal-processing computer that is used to calculate the required phase maps.

It is clear from Eq. (3) that the secondary fringe pattern of the ESPI is obtained by a subtraction process. Squaring Eq. (3) leads to

$$
I_{F}^{2}=16 I_{1} I_{2}\left[\sin ^{2}\left(\frac{\Delta \Phi}{2}\right) \sin ^{2}\left(\frac{\Delta \Phi}{2}+\Phi_{R}-\Phi_{B}\right)\right] .
$$


The second sine-squared term in Eq. (10) possesses the phase $\left(\Phi_{R}-\Phi_{B}\right)$ of the speckle pattern before deformation. The phase is generated by interfering two scattering light beams from the object and the reference surfaces. The phase term $\left(\Phi_{R}-\Phi_{B}\right)$ is restricted at every independent speckle area, and $\Phi_{R}$ and $\Phi_{B}$ are independent from each other. In addition, the optical system cannot record the interference fringes in one speckle region or the system must be stopped down. The term $\sin ^{2}\left[(\Delta \Phi / 2)+\Phi_{R}-\Phi_{B}\right]$ of Eq. (10) in one speckle is not correlated to other speckles; i.e., it is random in nature across the whole area. More specifically, no interference fringe can be generated from $\sin ^{2}\left[(\Delta \Phi / 2)+\Phi_{R}-\Phi_{B}\right]$ across the whole area. In other words, observed across the whole area to reveal the ensemble average behavior, only the term $\sin ^{2}(\Delta \Phi / 2)$ will show interference behavior across the whole picture. More specifically, the speckle output intensity recorded by the acquisition device in the optical system will not possess interference fringes generated within each speckle.

Since $\left(\Phi_{R}-\Phi_{B}\right)$ changes rapidly across the speckle patterns, the ensemble average of the second sinesquared term in Eq. (10) across the whole measurement area leads to

$$
\begin{aligned}
\left\langle\sin ^{2}\left(\frac{\Delta \Phi}{2}+\Phi_{R}-\Phi_{B}\right)\right\rangle \approx & \frac{1}{2 n \pi} \int_{0}^{2 n \pi} \sin ^{2}\left(\frac{\Delta \Phi}{2}+\Phi_{R}\right. \\
& \left.-\Phi_{B}\right) \mathrm{d}\left(\frac{\Delta \Phi}{2}+\Phi_{R}-\Phi_{B}\right) \\
= & \frac{1}{2} .
\end{aligned}
$$

Substituting Eq. (10) into Eq. (11) yields

$$
I_{F}^{2}=8 I_{1} I_{2}\left[\sin ^{2}\left(\frac{\Delta \Phi}{2}\right)\right]=4 I_{1} I_{2}[1-\cos (\Delta \Phi)] .
$$

Based on the Eq. (12) technique, the phase difference $\Delta \Phi$ can be obtained by adopting phase-shifting technology. The two phase-shifting algorithms developed with Eq. (12) as the base, the $(1,5)$ and the $(5,1)$ algorithm, are discussed here.

\section{A. $(1,5)$ Algorithm}

As mentioned above, the $(1,5)$ algorithm receives its name from procedures to generate the phase map; i.e., it requires one intensity map from the undeformed state and five intensity maps from the deformed state. The single intensity map recorded from the undeformed state is shown in Eq. (1). In addition, the five intensity maps from the deformed state are

$$
\begin{aligned}
& I_{A a}=I_{1}+I_{2}+2\left(I_{1} I_{2}\right)^{1 / 2} \cos \left(\Phi_{R}-\Phi_{B}+\Delta \Phi-2 \beta\right), \\
& I_{A b}=I_{1}+I_{2}+2\left(I_{1} I_{2}\right)^{1 / 2} \cos \left(\Phi_{R}-\Phi_{B}+\Delta \Phi-\beta\right), \\
& I_{A c}=I_{1}+I_{2}+2\left(I_{1} I_{2}\right)^{1 / 2} \cos \left(\Phi_{R}-\Phi_{B}+\Delta \Phi\right), \\
& I_{A d}=I_{1}+I_{2}+2\left(I_{1} I_{2}\right)^{1 / 2} \cos \left(\Phi_{R}-\Phi_{B}+\Delta \Phi+\beta\right),
\end{aligned}
$$

$$
I_{A e}=I_{1}+I_{2}+2\left(I_{1} I_{2}\right)^{1 / 2} \cos \left(\Phi_{R}-\Phi_{B}+\Delta \Phi+2 \beta\right),
$$

The square of the difference between Eqs. (13) and (1) is

$$
\begin{aligned}
I_{a}{ }^{\prime}= & \left(I_{B}-I_{A a}\right)^{2} \\
= & \left\{2 ( I _ { 1 } I _ { 2 } ) ^ { 1 / 2 } \left[\cos \left(\Phi_{R}-\Phi_{B}\right)-\cos \left(\Phi_{R}-\Phi_{B}\right.\right.\right. \\
& +\Delta \Phi-2 \beta)]\}^{2} \\
= & 8 I_{1} I_{2}\left[\sin ^{2}\left(\frac{\Delta \Phi}{2}-\beta\right)\right] \\
= & 4 I_{1} I_{2}[1-\cos (\Delta \Phi-2 \beta)], \\
I_{b}{ }^{\prime}= & \left(I_{B}-I_{A b}\right)^{2}=4 I_{1} I_{2}[1-\cos (\Delta \Phi-\beta)], \\
I_{c}{ }^{\prime}= & \left(I_{B}-I_{A c}\right)^{2}=4 I_{1} I_{2}[1-\cos (\Delta \Phi)], \\
I_{d^{\prime}}= & \left(I_{B}-I_{A d}\right)^{2}=4 I_{1} I_{2}[1-\cos (\Delta \Phi+\beta)], \\
I_{e}{ }^{\prime}= & \left(I_{B}-I_{A e}\right)^{2}=4 I_{1} I_{2}[1-\cos (\Delta \Phi+2 \beta)],
\end{aligned}
$$

Unlike the traditional phase-shifting techniques shown in Eq. (9), the phase difference $\Delta \Phi$ can be computed as

$$
\tan (\Delta \Phi)=\frac{[1-\cos (2 \beta)]}{\sin (\beta)} \frac{\left(I_{b}{ }^{\prime}-I_{d}{ }^{\prime}\right)}{\left(2 I_{c}{ }^{\prime}-I_{a}{ }^{\prime}-I_{e}{ }^{\prime}\right)} .
$$

\section{B. $(5,1)$ Algorithm}

The $(5,1)$ algorithm demands five intensity maps from the undeformed state and a single intensity map from the deformed state. The five intensity maps from the undeformed state are

$$
\begin{aligned}
I_{B a} & =I_{1}+I_{2}+2\left(I_{1} I_{2}\right)^{1 / 2} \cos \left(\Phi_{R}-\Phi_{B}-2 \beta\right) \\
& =I_{1}+I_{2}+2\left(I_{1} I_{2}\right)^{1 / 2} \cos \left(\Phi^{\prime}-\Delta \Phi-2 \beta\right), \\
I_{B b} & =I_{1}+I_{2}+2\left(I_{1} I_{2}\right)^{1 / 2} \cos \left(\Phi^{\prime}-\Delta \Phi-\beta\right), \\
I_{B c} & =I_{1}+I_{2}+2\left(I_{1} I_{2}\right)^{1 / 2} \cos \left(\Phi^{\prime}-\Delta \Phi\right), \\
I_{B d} & =I_{1}+I_{2}+2\left(I_{1} I_{2}\right)^{1 / 2} \cos \left(\Phi^{\prime}-\Delta \Phi+\beta\right), \\
I_{B e} & =I_{1}+I_{2}+2\left(I_{1} I_{2}\right)^{1 / 2} \cos \left(\Phi^{\prime}-\Delta \Phi+2 \beta\right),
\end{aligned}
$$

where $\Phi^{\prime}=\Phi_{R}-\Phi_{B}+\Delta \Phi$. In addition, the single intensity map from the deformed state can be rewritten from Eq. (2) as

$$
I_{A}=I_{1}+I_{2}+2\left(I_{1} I_{2}\right)^{1 / 2} \cos \left(\Phi^{\prime}\right) .
$$

The square of the difference between Eq. (17) and Eqs. (16) is

$$
\begin{aligned}
I_{a}^{\prime \prime} & =\left(I_{A}-I_{B a}\right)^{2} \\
& =\left\{2\left(I_{1} I_{2}\right)^{1 / 2}\left[\cos \left(\Phi^{\prime}\right)-\cos \left(\Phi^{\prime}-\Delta \Phi-2 \beta\right)\right]\right\}^{2} \\
& =8 I_{1} I_{2}\left[\sin ^{2}\left(\frac{\Delta \Phi}{2}-\beta\right)\right] \\
& =4 I_{1} I_{2}[1-\cos (\Delta \Phi-2 \beta)], \\
I_{b}^{\prime \prime} & =\left(I_{A}-I_{B b}\right)^{2}=4 I_{1} I_{2}[1-\cos (\Delta \Phi-\beta)], \\
I_{c}^{\prime \prime} & =\left(I_{A}-I_{B c}\right)^{2}=4 I_{1} I_{2}[1-\cos (\Delta \Phi)],
\end{aligned}
$$




$$
\begin{aligned}
I_{d}^{\prime \prime} & =\left(I_{A}-I_{B d}\right)^{2}=4 I_{1} I_{2}[1-\cos (\Delta \Phi+\beta)], \\
I_{e}{ }^{\prime \prime} & =\left(I_{A}-I_{B e}\right)^{2}=4 I_{1} I_{2}[1-\cos (\Delta \Phi+2 \beta)],
\end{aligned}
$$

Thus the phase difference $\Delta \Phi$ can be calculated as

$$
\tan (\Delta \Phi)=\frac{[1-\cos (2 \beta)]}{\sin (\beta)} \frac{\left(I_{b}{ }^{\prime \prime}-I_{d}{ }^{\prime \prime}\right)}{\left(2 I_{c}{ }^{\prime \prime}-I_{a}{ }^{\prime \prime}-I_{e}{ }^{\prime \prime}\right)} .
$$

Compared with the traditional phase-shifting techniques for ESPI, these newly developed algorithms shorten the measurement time effectively by reducing the numbers of the required intensity maps and by simplifying the calculation process. In addition, it is clear that the $(5,1)$ algorithm makes high-speed dynamic ESPI measurement feasible because only a single intensity map is needed from the deformed state.

\section{DC-(5,1) Algorithm}

To enhance further the capability of the ESPI and to extend the robustness of the $(5,1)$ algorithm mentioned above, a phase-analysis method that is further improved is presented in this section. This phaseanalysis method integrates the $(5,1)$ algorithm and the direct-correlation algorithm. ${ }^{6-8}$ Since a directcorrelation algorithm has been known to retrieve a phase map effectively from the noise speckle pattern by using the correlation coefficient, the advantages of integrating the $(5,1)$ algorithm and the directcorrelation algorithm when measurement data show strong speckle noise are obvious.

The correlation function of any two random variables, $X$ and $Y$, can be defined as

$$
\Gamma_{X Y}=\frac{\langle X Y\rangle-\langle X\rangle\langle Y\rangle}{\sigma_{X} \sigma_{Y}},
$$

where the \langle\rangle indicates the ensemble average, $\Gamma_{X Y}$ is the correlation coefficient, $\sigma_{X}=\left(\left\langle X^{2}\right\rangle-\langle X\rangle^{2}\right)^{1 / 2}$, and $\sigma_{Y}=\left(\left\langle Y^{2}\right\rangle-\langle Y\rangle^{2}\right)^{1 / 2}$.

For the case in which $X$ and $Y$ are independent, the following holds,

$$
\langle X Y\rangle=\langle X\rangle\langle Y\rangle,
$$

and this leads to a zero correlation coefficient between $X$ and $Y$.

From Eq. (20) the correlation coefficient of $I_{B}$ and $I_{A}$ as defined by Eqs. (1) and (2) can be rewritten as

$$
\Gamma_{A B}=\frac{\left\langle I_{B} I_{A}\right\rangle-\left\langle I_{B}\right\rangle\left\langle I_{A}\right\rangle}{\left(\left\langle I_{B}^{2}\right\rangle-\left\langle I_{B}\right\rangle^{2}\right)^{1 / 2}\left(\left\langle I_{A}^{2}\right\rangle-\left\langle I_{A}\right\rangle^{2}\right)^{1 / 2}} .
$$

As $I_{1}, I_{2}$, and $\Phi$ are mutually independent variables, each of the average values could be determined independently. When $\left\langle I_{1}\right\rangle=\left\langle I_{2}\right\rangle=\langle I\rangle$ is set, Eq. (22) can be simplified to

$$
\Gamma_{A B}=\frac{1}{2}[1+\cos (\Delta \Phi)] .
$$

If $\left\langle I_{1}\right\rangle=r\left\langle I_{2}\right\rangle$, then

$$
\Gamma_{A B}=\frac{1+r^{2}+2 r \cos (\Delta \Phi)}{(1+r)^{2}} .
$$

The correlation coefficient of each pixel can be obtained approximately by substituting $\Gamma$ into the expression for Pearson's coefficient of correlation shown below. ${ }^{9}$ More specifically, assuming that the intensities of the $m$ neighboring pixels before deformation is $a_{k}$ and the intensities of the $m$ neighboring pixels after deformation is $b_{k}$, Eq. (22) can be written as

$$
\begin{aligned}
\Gamma_{a b} & =\frac{\frac{1}{m} \sum^{m}\left(a_{k} b_{k}\right)-\left[\frac{1}{m} \sum^{m}\left(a_{k}\right)\right]\left[\frac{1}{m} \sum^{m}\left(b_{k}\right)\right]}{\sigma_{a} \sigma_{b}}, \\
\sigma_{a} & =\left\{\frac{1}{m} \sum^{m}\left(a_{k}{ }^{2}\right)-\left[\frac{1}{m} \sum^{m}\left(a_{k}\right)\right]^{2}\right\}^{1 / 2}, \\
\sigma_{b} & =\left\{\frac{1}{m} \sum^{m}\left(b_{k}{ }^{2}\right)-\left[\frac{1}{m} \sum^{m}\left(b_{k}\right)\right]^{2}\right\}^{1 / 2},
\end{aligned}
$$

where $\Gamma$ is the correlation coefficient for the central pixel. Equation (25a) is the well-known Pearson's coefficient of correlation for finite discrete sets of data and is taken here to be a local approximation to the coefficient of correlation of each pixel. The correlation coefficient for each pixel of the map can be computed by using Eqs. (25). The above-mentioned procedure leads to clear interference fringes because noise is reduced through the average process in the computation of the correlation coefficient. Substituting the correlation coefficients into Eq. (23) leads to the phases of each pixel.

The phase solved from Eq. (23) is modulated at $[0, \pi]$. To solve the phase correctly, whether or not the phase difference between the neighboring pixels is greater than $\pi$ must be determined in deciding the path of phase unwrapping. Noise will propagate in this process because it is path dependent. ${ }^{9-13}$ To overcome this drawback, the $(5,1)$ algorithm can be integrated with the direct-correlation algorithm to prevent the error from propagating. With the concepts revealed in the $(5,1)$ and $(1,5)$ algorithms, phase shifting can be executed before or after the object is deformed. The theoretical procedures needed to integrate the $(5,1)$ algorithms with the directcorrelation algorithm, which is termed the DC- $(5,1)$ algorithm, is obtained as follows. Similarly, the DC$(1,5)$ algorithm, which is obtained by integrating the $(1,5)$ algorithms with the direct-correlation algorithm, is also discussed.

\section{A. DC- $(1,5)$ Algorithm}

Instead of simply performing a subtraction and squaring operation in the $(1,5)$ algorithm, the DC$(1,5)$ algorithm is used to compute the correlation coefficient between the single intensity map from the undeformed state and the five intensity maps from 


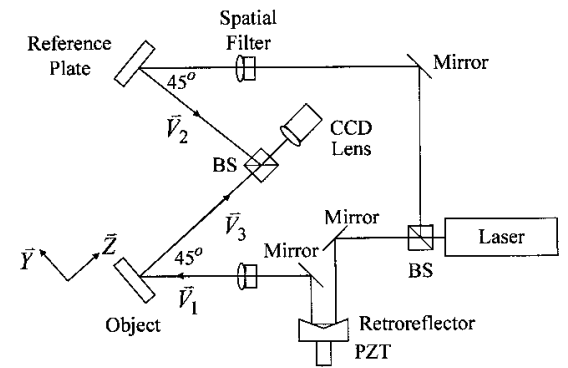

Fig. 1. ESPI optical arrangement adopted to demonstrate the new algorithms: BS, beam splitters.

the deformed state to enhance the noise immunity of the ESPI measurements. More specifically, the intensities of the undeformed object and the deformed object that correspond to each phase-shifting step are

$$
\begin{aligned}
I_{B} & =I_{1}+I_{2}+2\left(I_{1} I_{2}\right)^{1 / 2} \cos \left(\Phi_{R}-\Phi_{B}\right), \\
I_{A a} & =I_{1}+I_{2}+2\left(I_{1} I_{2}\right)^{1 / 2} \cos \left(\Phi_{R}-\Phi_{B}+\Delta \Phi-2 \beta\right), \\
I_{A b} & =I_{1}+I_{2}+2\left(I_{1} I_{2}\right)^{1 / 2} \cos \left(\Phi_{R}-\Phi_{B}+\Delta \Phi-\beta\right), \\
I_{A c} & =I_{1}+I_{2}+2\left(I_{1} I_{2}\right)^{1 / 2} \cos \left(\Phi_{R}-\Phi_{B}+\Delta \Phi\right), \\
I_{A d} & =I_{1}+I_{2}+2\left(I_{1} I_{2}\right)^{1 / 2} \cos \left(\Phi_{R}-\Phi_{B}+\Delta \Phi+\beta\right), \\
I_{A e} & =I_{1}+I_{2}+2\left(I_{1} I_{2}\right)^{1 / 2} \cos \left(\Phi_{R}-\Phi_{B}+\Delta \Phi+2 \beta\right),
\end{aligned}
$$

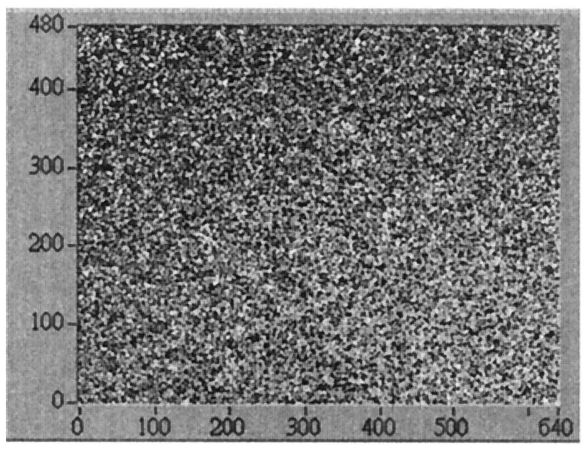

(a)

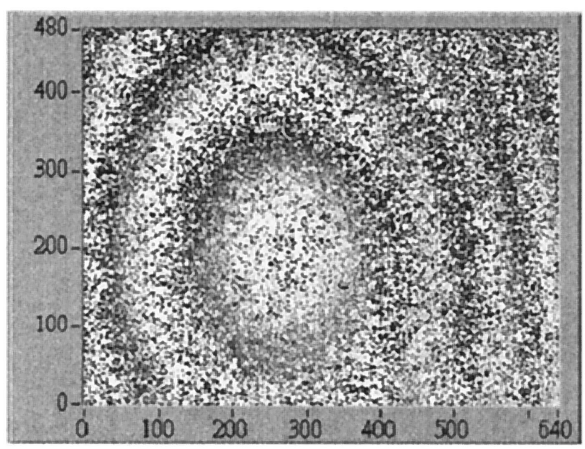

(c)
Furthermore the correlation coefficient for each phase-shifting step can be rewritten by use of Eq. (22) as

$$
\begin{aligned}
& \Gamma_{a}=\frac{1}{2}[1+\cos (\Delta \Phi-2 \beta)], \\
& \Gamma_{b}=\frac{1}{2}[1+\cos (\Delta \Phi-\beta)], \\
& \Gamma_{c}=\frac{1}{2}[1+\cos (\Delta \Phi)], \\
& \Gamma_{d}=\frac{1}{2}[1+\cos (\Delta \Phi+\beta)], \\
& \Gamma_{e}=\frac{1}{2}[1+\cos (\Delta \Phi+2 \beta)] ;
\end{aligned}
$$

i.e., $\Gamma_{a}$ to $\Gamma_{e}$ are the five coefficients of correlation between $I_{B}$ and $I_{A a}$ to $I_{A e}$, respectively. The information of these five correlation coefficients are related to the correct phase $\Delta \Phi$ by

$$
\tan (\Delta \Phi)=\frac{[1-\cos (2 \beta)]}{\sin (\beta)} \frac{\left(\Gamma_{b}-\Gamma_{d}\right)}{\left(2 \Gamma_{c}-\Gamma_{a}-\Gamma_{e}\right)} .
$$

With the proper signs of the numerator and the denominator, Eq. (28) leads to the phases in $[0,2 \pi]$, which can then be integrated with the phaseunwrapping technique ${ }^{14}$ to retrieve the deformation information.

\section{B. DC- $(5,1)$ Algorithm}

The steps needed to integrate the $(5,1)$ algorithm with the direct-correlation algorithm are examined

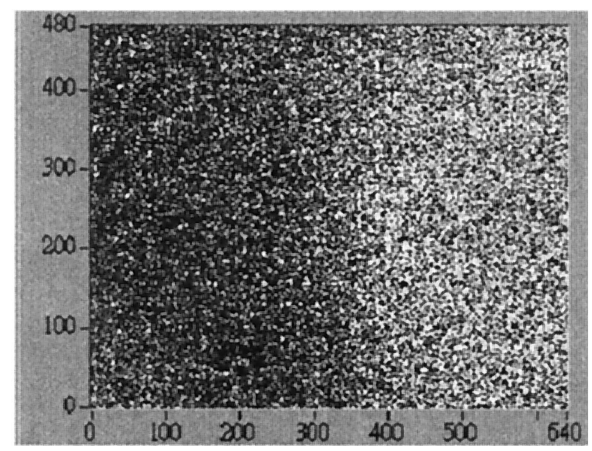

(b)

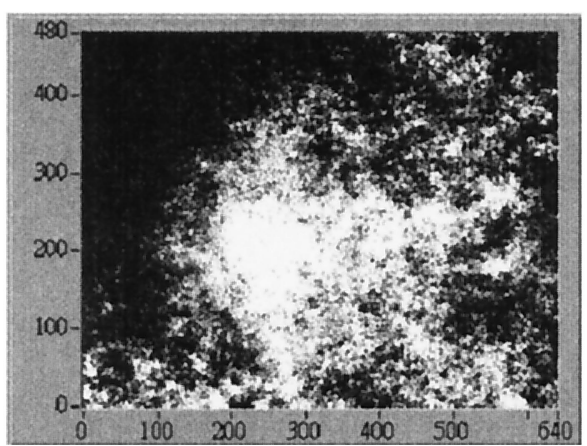

(d)

Fig. 2. Measurement results from using traditional phase-shifting technology. 


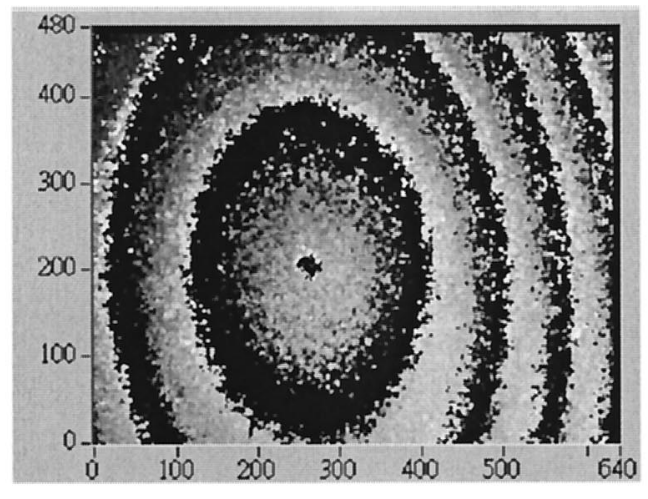

(a)

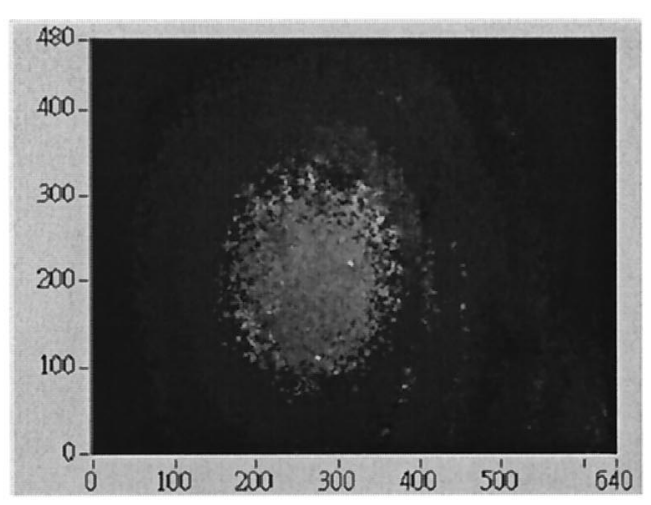

(b)

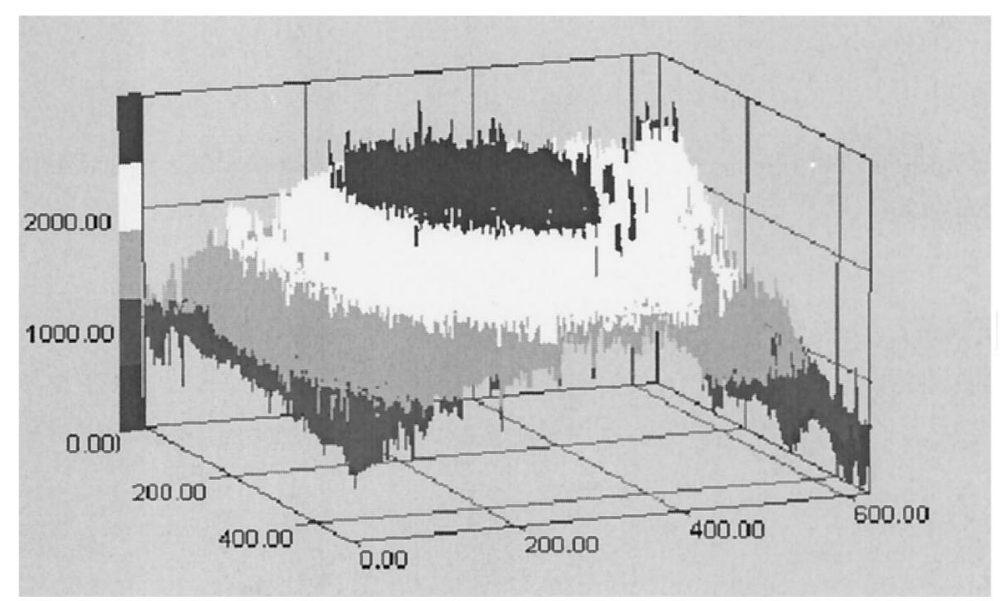

(c)

Fig. 3. Experimental results retrieved from the $(5,1)$ algorithm.

herein. Similar to the DC-(1,5) algorithm, the DC$(5,1)$ algorithm performs the correlation calculation between the five intensity maps from the original state and the single intensity from the deformed state. The noise immunity of the direct-correlation algorithm can be maintained in this algorithm. In addition, the advantages of the $(5,1)$ algorithm such as its applicability for high-speed dynamic ESPI measurements are maintained as well. Starting from the intensity maps recorded before and after the specimen is deformed, i.e.,

$$
\begin{aligned}
I_{B a} & =I_{1}+I_{2}+2\left(I_{1} I_{2}\right)^{1 / 2} \cos \left(\Phi^{\prime}-\Delta \Phi-2 \beta\right), \\
I_{B b} & =I_{1}+I_{2}+2\left(I_{1} I_{2}\right)^{1 / 2} \cos \left(\Phi^{\prime}-\Delta \Phi-\beta\right), \\
I_{B c} & =I_{1}+I_{2}+2\left(I_{1} I_{2}\right)^{1 / 2} \cos \left(\Phi^{\prime}-\Delta \Phi\right), \\
I_{B d} & =I_{1}+I_{2}+2\left(I_{1} I_{2}\right)^{1 / 2} \cos \left(\Phi^{\prime}-\Delta \Phi+\beta\right), \\
I_{B e} & =I_{1}+I_{2}+2\left(I_{1} I_{2}\right)^{1 / 2} \cos \left(\Phi^{\prime}-\Delta \Phi+2 \beta\right), \\
I_{A} & =I_{1}+I_{2}+2\left(I_{1} I_{2}\right)^{1 / 2} \cos \left(\Phi^{\prime}\right),
\end{aligned}
$$

we can rewrite the correlation coefficients for each phase-shifting step as

$$
\Gamma_{a}^{\prime}=\frac{1}{2}[1+\cos (\Delta \Phi-2 \beta)]
$$

$$
\begin{aligned}
\Gamma_{b}{ }^{\prime} & =\frac{1}{2}[1+\cos (\Delta \Phi-\beta)], \\
\Gamma_{c}{ }^{\prime} & =\frac{1}{2}[1+\cos (\Delta \Phi)], \\
\Gamma_{d}{ }^{\prime} & =\frac{1}{2}[1+\cos (\Delta \Phi+\beta)], \\
\Gamma_{e}{ }^{\prime} & =\frac{1}{2}[1+\cos (\Delta \Phi+2 \beta)] .
\end{aligned}
$$

The phase difference $\Delta \Phi$ can then be calculated by

$$
\tan (\Delta \Phi)=\frac{[1-\cos (2 \beta)]}{\sin (\beta)} \frac{\left(\Gamma_{b}^{\prime}-\Gamma_{d}^{\prime}\right)}{\left(2 \Gamma_{c}^{\prime}-\Gamma_{a}^{\prime}-\Gamma_{e}^{\prime}\right)}
$$

Implementing the above-mentioned phase-shifting 14 and signal-processing algorithms for real experimental data is examined in Section 5.

\section{Experimental Verification and Results}

The optical setup used for the experimental verifications is shown in Fig. 1, which shows a typical ESPI setup, except for the configuration used to execute the phase-shifting operations. In a typical phaseshifting operation a mirror driven by some sort of actuator capable of inducing small displacement is used to induce the predesignated phase needed. 


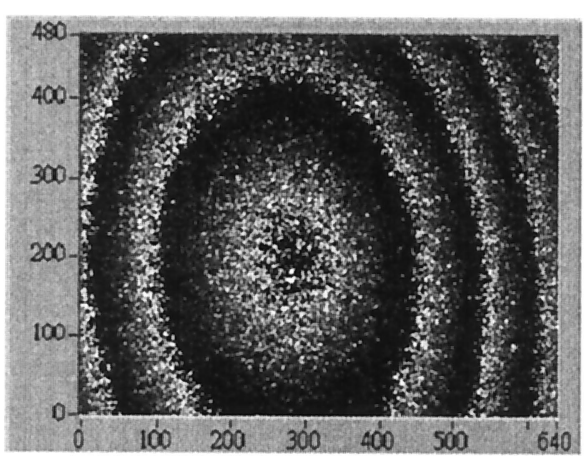

(a)

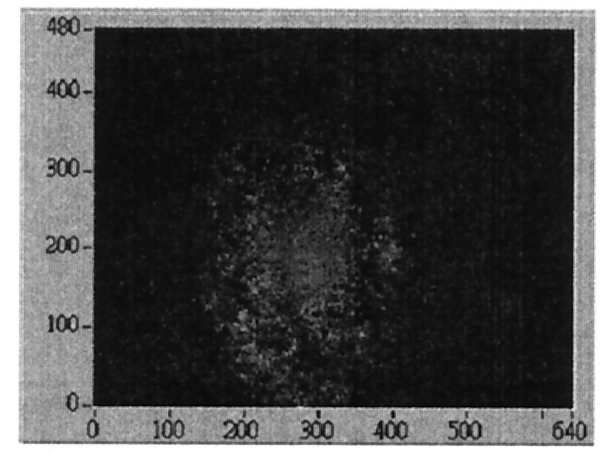

(b)

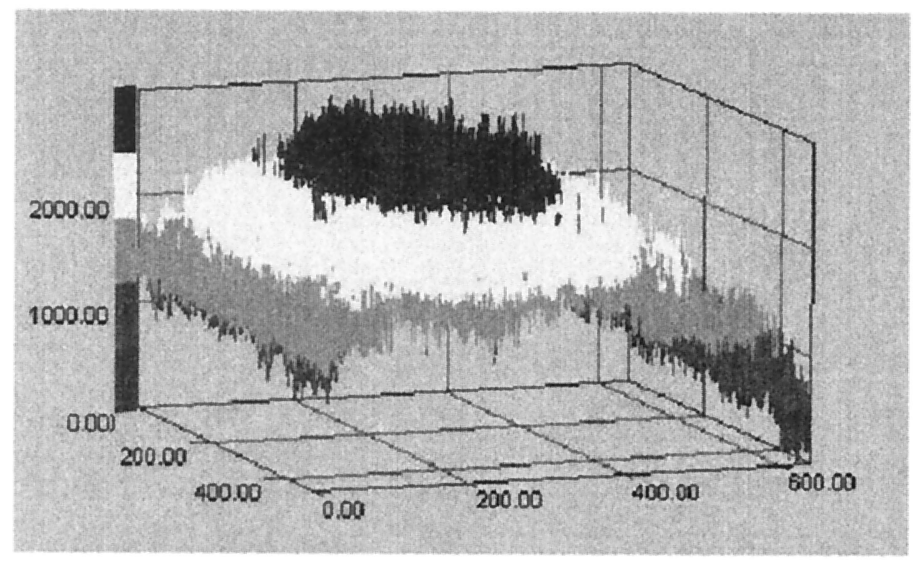

(c)

Fig. 4. Experimental results computed from the DC-(5,1) algorithm.

In the configuration shown in Fig. 1 a lead zirconate titanate, PZT, actuator from Tokin Corpora$\operatorname{tion}^{15,16}$ is attached to a fix end to drive a retroreflector to perform the phase-shifting procedures. The main advantage of the configuration shown is that the optical path does not change during the phase-shifting operation, which can significantly reduce efforts needed to set up the spatial filters typically used within the ESPI optical path. The phase obtained from this arrangement can be written as

$$
\begin{aligned}
\Delta \Phi= & \left(\mathbf{K}_{3}-\mathbf{K}_{1}\right) \cdot \mathbf{L} \\
= & \left(\mathbf{V}_{3}-\mathbf{V}_{1}\right) \cdot \mathbf{L} \\
= & \frac{2 \pi}{\lambda}\left\{\hat{Z}-\left[\sin \left(45^{\circ}\right) \hat{Y}-\cos \left(45^{\circ}\right) \hat{Z}\right]\right\} \cdot\left[L_{X} \hat{X}\right. \\
& \left.+L_{Y} \hat{Y}+L_{Z} \hat{Z}\right] \\
= & \frac{2 \pi}{\lambda}\left\{\left[1+\cos \left(45^{\circ}\right)\right] L_{Z}-\sin \left(45^{\circ}\right) L_{Y}\right\},
\end{aligned}
$$

where $\mathbf{L}$ is the displacement vector and $\mathbf{L}_{Y}$ and $\mathbf{L}_{Z}$ are the displacements along the $Y$ and the $Z$ axes. This arrangement was used to perform a five-step phaseshifting algorithm to measure the displacement of an object under a point load. The phase maps from the undeformed and the deformed states, i.e., a total of 10 intensity maps, were recorded. These data were first computed by using both the $(5,1)$ and the $(1,5)$ algorithms to compare results generated by these two algorithms. Furthermore phase-unwrapping methods called the discrete cosine transform (DCT) (Ref. 9) and the discrete Fourier transform (DFT) (Ref. 14) were adopted for comparison purposes owing to their path independency and simplification.

Some issues related to the domain of interest about DCT and DFT must be further explained owing to their behaviors at the boundaries. ${ }^{10,11,14}$ Considering the case that $N \times N$ sample points must be analyzed, a $N \times N$ matrix that represents the phase change induced by the displacement are to be obtained. The DCT algorithm can be executed across the $N \times N$ sample points directly. On the other hand, to use the DFT algorithm, the $N \times N$ data region must be expanded to $2 N \times 2 N$ by taking the mirror reflection of the original $N \times N$ data points and then stitched back. More specifically, the concept of period normal equations and the selfrepeating behavior of the DFT would induce a jump on the boundary unless the methodology mentioned above was implemented. Furthermore, the fast Fourier transform (FFT) was also adopted, because adopting DFT increases the number of data points that must be handled by a factor of 4 . To meet the 
need of FFT, the domain was further edited by adding zeros to change the $2 N \times 2 N$ to $2^{m} \times 2^{m}$, where $m$ is an exponent of a power of 2 . It has long been known that the FFT runs more than 4 times faster than the data points to be handled. The results from using the traditional phase-shifting technology mentioned above are shown in Fig. 2. Figure 2(a) shows the phase map from the undeformed state computed by the traditional five-step phase-shifting technique. Figure 2(b) is the phase map of the deformed state computed by the five-step phase-shifting technique as well. Subtracting Fig. 2(b) from Fig. 2(a) generated the results shown in Fig. 2(c). Figure 2(d) is the phase-unwrapping results obtained from the DCT. The noisy and the cloudy results shown in Fig. 2 clearly reveal that the traditional phase-shifting technique is not adequate to handle such a noisy data set. The traditional technique not only requires a long measurement time but also generates unnecessary data sets. It is clear from the results above that the traditional phase-shifting approach is not suitable for noisy ESPI measurement data.

Figure 3 was obtained by using the $(5,1)$ algorithm and the DCT phase-unwrapping algorithm to compute the same data set. Figure 3(a) is the phase map computed by the $(5,1)$ algorithm. Figure $3(\mathrm{~b})$ is the unwrapped phase obtained by the DCT. Figure 3 (c) is the three-dimensional plot of the data shown in Fig. 3(b). Because only a one phase-shifting procedure needs to be performed in the $(5,1)$ algorithm, the measurement time is significantly reduced. Note that more intensity maps usually introduce more noise due to an external disturbance such as air turbulence or outside vibration. The results from using the $(1,5)$ algorithm were found to be similar to those data in Fig. 3 and thus are not discussed here. A comparison of the results in Figs. 2 and 3 indicates that the quality of the phase-unwrapping result improves with adoption of the $(5,1)$ algorithm.

Figure 4 shows the result from combining the $(5,1)$ algorithm and the direct-correlation algorithm, i.e., the DC- $(5,1)$ algorithm. Figure 4(a) shows the wrapped phase map obtained with this integrated algorithm. Figure 4(b) shows the unwrapped phase computed from using the DCT, and Fig. 4(c) shows the three-dimensional plot of the data in Fig. 4(b). Again the results from using the DC-(1,5) algorithm are very similar to those data in Fig. 4.

By comparing the results in Fig. 4 and those in Fig. 3 , it is clear that the DC- $(5,1)$ algorithm provides higher-quality ESPI phase maps than the $(5,1)$ algorithm. Note that the only disadvantage of the DC$(5,1)$ algorithm when compared with the $(5,1)$ algorithm is the longer computation time needed because more tedious calculation steps must be performed to compute the correlation coefficients.

\section{Conclusions and Discussions}

To overcome some difficulties, such as long measurements, long computation time, and noisy or cloudy phase maps, in using a traditional phase-shifting technique for ESPI and the like, two new set of meth- ods were developed. The first set of methods includes two similar algorithms, the $(5,1)$ algorithm and the $(1,5)$ algorithm. Both of these algorithms can reduce the phase-shifting steps needed in either the undeformed or the deformed state. Our experimental investigations indicate that these two approaches provide comparable experimental results. Nevertheless the $(5,1)$ algorithm appears to be more useful because a high-speed camera can be used to record the specimen ESPI intensity map while the specimen is undergoing deformation. This basic configuration extends the application range of the ESPI into a high-speed dynamic vibration regime. The two new approaches were created by integrating the direct-correlation algorithm with either the $(5,1)$ algorithm or the $(5,1)$ algorithm. Experimental results were also obtained to compare the effectiveness of all these newly developed signal-processing algorithms. It has been shown clearly that the $(5,1)$ algorithm can be executed faster than traditional approaches. In addition, owing to the generic features of the $(5,1)$ algorithm, this approach is suitable for high-speed dynamic deformation. To enhance further the effectiveness of the newly developed algorithms for ESPI applications, the $(5,1)$ algorithm was integrated with the direct-coefficient algorithm to form the DC- $(5,1)$ algorithm. For noisy phase maps the ESPI experimental results processed from using this novel DC- $(5,1)$ algorithm were clear and free from the noise-propagation problem.

\section{References and Notes}

1. R. Jones and C. Wykes, Holographic and Speckle Interferometry (Cambridge University, Cambridge, UK, 1983).

2. A. J. Moore and J. R. Tyrer, "Phase-stepped ESPI and moire interferometry for measuring the stress-intensity factor and $J$ integral," Exp. Mech. 35, 306-314 (1995).

3. J. N. Butters and J. A. Leendertz, "A double exposure technique for speckle pattern interferometry," J. Phys. E 4, 277279 (1971).

4. K. Creath, "Phase-shifting speckle interferometry," Appl. Opt. 24, 3053-3058 (1985).

5. P. Hariharan, B. F. Orbel, and T. Eiju, "Digital phase-shifting interferometry: a simple error-compensating phase calculation," Appl. Opt. 26, 2504-2507 (1987).

6. D. R. Schmitt and R. W. Hunt, "Optimization of fringe pattern calculation with direct correlations in speckle interferometry," Appl. Opt. 36, 8848-8857 (1997).

7. C. Gorecki, "Phase-correlation techniques for quasi-real-time measurement of deformations with digital speckle interferometry," Appl. Opt. 33, 2933-2938 (1994).

8. J. Pomarico, R. Arizaga, R. Towoba, and H. Rabal, "Algorithm to compute spacing of digital speckle correlation fringes," Optik (Stuttgart) 95, 125-127 (1993).

9. D. C. Ghiglia and L. A. Romero, "Robust two-dimensional weighted and unweighted phase unwrapping that uses fast transforms and iterative methods," J. Opt. Soc. Am. A 11, 107-117 (1994).

10. H. Takajo and T. Takahashi, "Noniterative method for obtaining the exact solution for the normal equation in a leastsquares phase estimation,” J. Opt. Soc. Am. A 5, 1818-1827 (1988).

11. H. Takajo and T. Takahashi, "Least-squares phase estimation from the phase difference," J. Opt. Soc. Am. A 5, 416-425 (1988). 
12. H. Y. Chang, C. W. Chen, C. K. Lee, and C. P. Hu, "The tapestry cellular automata phase unwrapping algorithm for nondestructive testing," J. Opt. Lasers Eng. 30, 487-502 (1998).

13. C. W. Chen, H. Y. Chang, and C. K. Lee, "An innovative phase shifting system for nondestructive testing," J. Chin. Soc. Appl. Mech. 14, 31-39 (1998).

14. Y. C. Chen, S. S. Lee, C. M. Lee, C. K. Lee, and G. B. Yeh, "New methodology for measuring highly aberrated wave fronts in- duced by diffractive optical elements," in Testing, Packaging, Reliability, and Applications of Semiconductor Lasers IV, M. Fallahi, J. Linden, and S. Wang, eds., Proc. SPIE 3626, 248 259 (1999).

15. S. Takahashi, "Multilayer piezoelectric ceramics actuators and their applications," Jpn. J. Appl Phys. 24, 41-45 (1985).

16. Tokin America Inc., "Multilayer piezoelectric actuator," Insp. Rec. 912-46S-02039, displacement: $14.4 \mu \mathrm{m}$ at $100 \mathrm{~V}$ dc for 17.981-mm length (1991). 